\title{
Áreas del conocimiento de enfermería en la calidad científica de la Revista Enfermería Universitaria de la ENEO 2007-2011
}

\author{
Rebeca Esquivel-Escobar, * Lasty Balseiro-Almario, ** Federico Sacristán-Ruíz,*** Gandhy Ponce-Gómez, **** \\ Silvia Hernández-Rosas*****
}

\author{
RESUMEN
}

El problema de estudio que se planteó fue: ¿cuáles son las áreas del conocimiento de la enfermería en la evaluación de la calidad científica de la Revista Enfermería Universitaria teniendo como base los indicadores bibliométricos empleados durante el periodo 2007-2011? Marco teórico: La revista científica tiene como función, además de la certificación de la ciencia, establecer e implementar los criterios de calidad que se utilizan para la realización y divulgación de las investigaciones, así como ayudar a consolidar las áreas de investigación, constituirse en el depósito de las informaciones de interés internacionales, nacional o regional y entrenar revisores y autores en análisis y crítica, mejorando la calidad de la ciencia. Material y métodos: La variable medida fue: las áreas del conocimiento de enfermería en la Revista Enfermería Universitaria. El diseño metodológico fue de corte cuantitativo, no experimental, transversal, retrospectivo, comparativo, analítico, descriptivo y diagnóstico. La muestra de estudio fue el total de artículos de la revista editados en los años 2007-2011. Resultados: En las áreas de apoyo del conocimiento de enfermería en los aspectos de enfermería comunitaria y de administración de los servicios de enfermería se publicaron 15 artículos en cada rubro. Conclusiones: En la Revista Enfermería Universitaria las áreas temáticas del conocimiento de enfermería que predominan son las de los artículos de aspectos metodológicos de la profesión, administración de los servicios de enfermería y enfermería comunitaria.

Palabras clave: Evaluación, indicadores bibliométricos, áreas del conocimiento, enfermería.

\section{Nursing knowledge areas in scientific quality of the ENEO's Enfermería Universitaria J ournal from 2007-2011}

\begin{abstract}
The study question is based on nursing knowledge areas analysis in scientific quality assessment of the ENEO's Enfermería Universitaria Journal scientific quality during the period 2007-2011. Theoretical framework: Among the journal functions are to establish health criteria for the development and publishing of research works, to help strengthen the areas of research, to become a reservoir of information of national and international interest, to train authors and reviewers in analysis and critic, all to include the quality of the science. Materials and methods: The measured variable of interest was the ENEO's Enfermería Universitaria Journal scientific and the areas of nursing knowledge were assessed. The methodological
\end{abstract}

\footnotetext{
* Pasante de la Licenciatura en Enfermería y Obstetricia de la ENEO-UNAM.

** Profesor de Carrera Titular "B" de la ENEO-UNAM. Responsable del proyecto.

*** Profesor Asociado "C" de la ENEO-UNAM. Corresponsable del proyecto. **** Colaboradores del proyecto.
}

Recibido para publicación: 04/04/2014. Aceptado: 06/05/2014.

Correspondencia: Rebeca Esquivel-Escobar. E-mail: rbeses@hotmail.com

Este artículo puede ser consultado en versión completa en http://www.medigraphic.com/enfermerianeurologica 
design was quantitative, not experimental, transversal, retrospective comparative, analytical, descriptive and diagnostic. The study sample was the total of issues published from 2007-2011. Results: In relation to the areas of support of nursing knowledge, there were 15 articles on communitarian nursing and 15 of nursing service management. Conclusions: The articles which is prevalent in the Enfermería Universitaria Journal are about methodological issues on care, service administration and communitarian nursing.

Key words: Assessment, bibliometric indicators, knowledge areas, nursing.

\section{INTRODUCCIÓN}

$\mathrm{E}$ 1 problema de estudio que se planteó fue conocer cuáles son las áreas del conocimiento de enfermería en la evaluación interna de la Revista Enfermería Universitaria, teniendo como base los indicadores bibliométricos durante el periodo 2007-2011. El objetivo fue evaluar por áreas la dimensión del conocimiento de enfermería durante los años 2007-2011 con base en los indicadores bibliométricos. El objetivo específico fue identificar los artículos publicados por áreas del conocimiento de enfermería. Marco teórico. Para Gálvez Toro y cols., ${ }^{1}$ la construcción de la ciencia es un proceso complejo en el que la publicación del producto de su actividad es un hecho culminante y el resultado final de la actividad científica. Sin embargo, este resultado es parte de un fenómeno más amplio denominado ciclo de la producción científica; según éste, la publicación de un producto científico es el inicio y condición necesaria para su difusión.

Así, para Lorenzini Erdmann y cols., ${ }^{2}$ el periódico científico es una publicación en serie que tiene una periodicidad definida. Éste también es designado como revista científica o técnico-científica. En el medio académico, se observa que hay una preferencia por la denominación de revista científica. Así, la revista científica tiene como función, además de la certificación de la ciencia, establecer e implementar los criterios de calidad para la realización y divulgación de investigaciones y ayudar a consolidar las áreas de investigación para constituirse en depósito de las informaciones de interés internacionales, nacional o regional, además de entrenar a los revisores y autores en el análisis y crítica, lo que mejora la calidad de la ciencia.

Así, para Serrano Gallardo y cols., ${ }^{3}$ la comunicación de la información científica es un rasgo esencial de la ciencia moderna y dar a conocer los hallazgos de las investigaciones es de crucial importancia para que se siga produciendo conocimiento científico; sin duda, las publicaciones periódicas son el vehículo por excelencia para este menester.

Para Araujo-Pulido y cols., ${ }^{4}$ las publicaciones científicas en el campo de la salud van cada día en aumento en lo que a número de revistas periódicas y artículos se refiere; por ello, es indispensable evaluar la calidad, repercusión y visibilidad de éstas en la comunidad científica para que puedan ser incluidas e indizadas en distintas bases de datos nacionales e internacionales.

La publicación de un artículo en una revista científica requiere de un proceso riguroso de revisión y validación para asegurar la calidad y el aporte de su contenido. De hecho, la evaluación de la calidad y de la repercusión de las revistas científicas constituye uno de los métodos más habitualmente empleados para obtener información sobre su impacto entre los miembros de la comunidad científica.

Para Pardo Serrano y cols..$^{5}$ la metodología bibliométrica constituye un instrumento esencial para evaluar el producto de la ciencia y, en el caso de disciplinas incipientes en materia de investigación como lo es la enfermería, esta disciplina se constituye como una herramienta esencial para determinar su avance y estado de consolidación, ya que permite analizar diferentes aspectos relativos a la producción, consumo de la información y repercusión de ésta. De hecho, para M. Sobrido Prieto y cols. ${ }^{6}$ los estudios bibliométricos permiten, entre otras cuestiones, evaluar la calidad de las revistas científicas y determinar el estado de la cuestión en una disciplina.

En la ciencia de la enfermería estos estudios son relativamente recientes, pues se iniciaron hace poco más de una década; se han publicado estudios relativos a las áreas concretas de la enfermería, como el realizado por Barreda y cols. en el área materno infantil o el llevado a cabo por Asiaín y Marín que está centrado en la enfermería intensiva. También se han publicado estudios sobre la producción enfermera a través de las revistas de mayor difusión; entre éstos se encuentra el realizado por Torra i Bou, quien analizó tres publicaciones enfermeras en el trienio 1991-1993. Gálvez Toro y cols., basándose en el análisis de referencias y de citas, llegaron a la conclusión de que las enfermeras consultan pocas revistas de enfermería al escribir sus artículos. En cuanto al área temática, existen artículos por ámbito que incluyen la asistencial, gestión, docencia e investigación; también de colaboración que incluyen un solo autor-coautoría y un número de firmas por trabajo; de área temática que incluye a la historia y fundamentos de enfermería, ética y legislación, enfermería psicosocial, salud mental, enfermería psiquiátrica, ecología humana, enfermería comunitaria y bioestadística y demografía, además de microbiología, salud pública, farmacología, nutrición y 
dietética, cuidados básicos, enfermería médico-quirúrgica, materno-infantil, geriatría y gerontología y administración en la revisión del área de especialidad de enfermería.

La Fundación Index ${ }^{7}$ considera que las áreas de especialidad de la enfermería son los cuidados intensivos, geriatría y gerontología, nefrología, urología, radiología, cardiología y obstetricia. Además existe el área lingüística, cultural y geográfica. Finalmente, para Gómez Caridad y Bordons Gangas $^{8}$ los resultados de la investigación pueden ser publicados, según su tema y alcance, en revistas nacionales o internacionales. Las publicaciones internacionales están escritas preferentemente en lengua inglesa y su destino es la comunidad científica internacional, mientras que las revistas nacionales, destinadas a una audiencia local, suelen estar en el idioma del país de origen. Podría decirse, entonces, que la ciencia básica es la ciencia "internacional" por excelencia y que, por tanto, tiende a transmitirse en revistas internacionales. Sin embargo, la ciencia aplicada, extendida como una explotación local de la ciencia básica, puede encontrar un buen vehículo de transmisión en las revistas de carácter nacional.

\section{MATERIAL Y MÉTODOS}

La variable medida correspondió a las áreas del conocimiento de la enfermería en calidad científica de la Revista Enfermería Universitaria. A esta dimensión se le consideraron los siguientes indicadores: cuidados básicos de enfermería, aspectos metodológicos, docencia, médico-quirúrgica, materno-infantil, historia y filosofía del cuidado, así como ética y legislación, bioética, ecología y demografía, ecología y salud laboral, enfermería comunitaria, administración de los servicios de enfermería y otros.

El diseño metodológico fue de corte cuantitativo no experimental, transversal, retrospectivo, comparativo, analítico, descriptivo y diagnóstico. La muestra de estudio fue el total de los artículos de la revista editados en los años 2007-2011, los cuales fueron un total de 148. Como criterio de inclusión se consideraron todos los números de la Revista Universitaria publicados en esos años. Se excluyeron todos los artículos de la revista publicados en los números anteriores al 2007 y posteriores al 2011.

En esta investigación ningún artículo, sección o número de la revista publicada en el periodo citado fue eliminado. Las técnicas consideradas en la evaluación interna fueron la observación y el análisis directo mediante una revisión detallada de cada una de las revistas. Para realizar el proceso de evaluación interna, se hizo un análisis exhaustivo de cada uno de los números de la revista, ordenando y clasificando los datos en una base diseñada previamente para la aplicación posterior del paquete estadístico SPSSV-14 que se utilizó para el análisis de la información.

En las consideraciones éticas no existió ningún riesgo para los pares y, desde luego, se tomó en cuenta el principio de anonimato y privacidad conforme al reglamento de la Ley General de Salud en materia de Investigación, título 2 capítulo XIII, XVI, C de la Secretaría de Salud, el cual se refiere al principio de la privacidad y en donde prevalece el criterio de respeto a la dignidad y a la protección de los derechos y bienestar de los seres humanos.

Los recursos utilizados en esta investigación fueron los humanos, con dos profesores de carrera de tiempo completo; uno titular "B" y dos asociados " $\mathrm{C}$ ", así como una pasante de la licenciatura en Enfermería y Obstetricia. Los recursos físicos y tecnológicos fueron los propios de la División de Estudios de Postgrado de la ENEO, específicamente de la Unidad de Investigación; finalmente el aspecto financiero fue cubierto en su totalidad por el propio grupo de investigación.

\section{RESULTADOS}

En cuanto a la dimensión de las áreas del conocimiento de la enfermería en cuidados básicos se refiere, fueron publicados 12 artículos; sobre los aspectos metodológicos de la profesión se publicaron 33 artículos y en docencia se pu-

Cuadro I. Artículos por áreas de conocimiento de enfermería: cuidados básicos.

\begin{tabular}{|c|c|c|c|c|c|c|c|c|c|c|c|c|}
\hline \multirow[b]{2}{*}{ Año } & \multicolumn{2}{|c|}{ Cuidados básicos } & \multicolumn{2}{|c|}{ Metodología } & \multicolumn{2}{|c|}{$\begin{array}{l}\text { Docencia en } \\
\text { enfermería }\end{array}$} & \multicolumn{2}{|c|}{ Médico QCA } & \multicolumn{2}{|c|}{ Materno infantil } & \multicolumn{2}{|c|}{$\begin{array}{l}\text { Historia y filosofía } \\
\text { del cuidado }\end{array}$} \\
\hline & FO & $\%$ & FO & $\%$ & $\mathrm{FO}$ & $\%$ & FO & $\%$ & FO & $\%$ & FO & $\%$ \\
\hline 2007 & 4 & 33.33 & 3 & 9.09 & 5 & 14.70 & 0 & 0 & 0 & 0 & 3 & 30 \\
\hline 2008 & 5 & 41.66 & 11 & 33.33 & 10 & 29.41 & 0 & 0 & 0 & 0 & 1 & 10 \\
\hline 2009 & 0 & 0 & 6 & 18.18 & 7 & 20.58 & 0 & 0 & 0 & 0 & 2 & 20 \\
\hline 2010 & 0 & 0 & 4 & 12.12 & 8 & 14.81 & 0 & 0 & 3 & 100 & 4 & 40 \\
\hline \multirow[t]{2}{*}{2011} & 3 & 25 & 9 & 27.27 & 4 & 11.76 & 0 & 0 & 0 & 0 & 0 & 0 \\
\hline & 12 & 100 & 33 & 100 & 34 & 100 & 0 & 0 & 3 & 100 & 10 & 100 \\
\hline
\end{tabular}

Fuente: Balseiro L. y cols. Evaluación interna de la Revista Enfermería Universitaria 2007-2011. México, 2013. 
blicaron 34 artículos; en médico-quirúrgica cero artículos, en materno-infantil tres artículos y en historia y filosofía del cuidado 10 artículos (Cuadro I).

Es posible observar, en estos resultados, que las áreas del conocimiento de mayor publicación son las metodológicas e instrumentales, las de docencia y las de cuidados básicos. Por el contrario, las áreas de materno infantil, médico-quirúrgica e historia y filosofía del cuidado quedan con poca o nula visibilidad en las publicaciones revisadas (Figura 1). En cuanto a las áreas de apoyo del conocimiento de enfermería en los aspectos de ética y legislación se publicaron cuatro artículos, en el área de bioética, cero artículos, en ecología y demografía, cero artículos, en salud laboral de enfermería siete artículos, en enfermería comunitaria 15 artículos, en administración de los servicios de enfermería 15 artículos y en otros de temá-

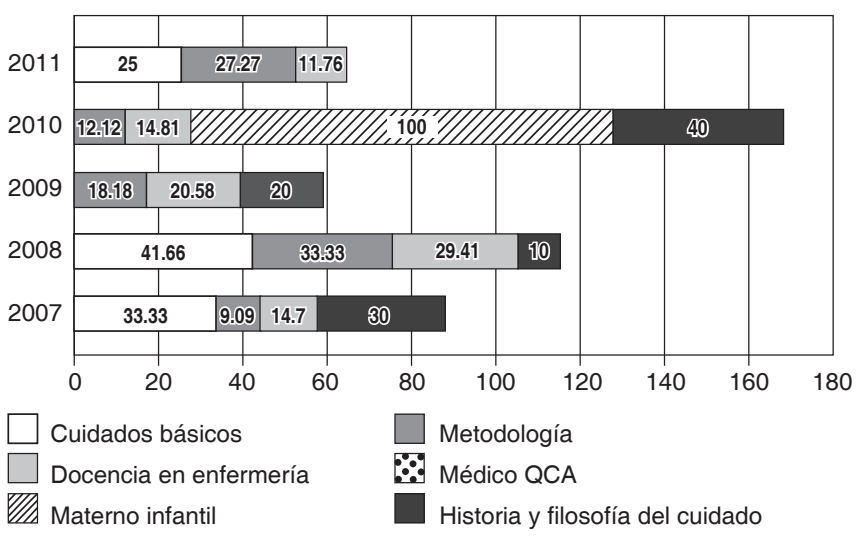

Figura 1. Cuidados básicos, metodología y docencia de enfermería. En esta figura se puede observar que el $42 \%$ de los artículos de cuidados básicos fueron publicados en el 2008, así como el 33\% de artículos sobre metodología de investigación y el $29.41 \%$ de artículos de docencia en enfermería en el mismo año. El 100\% de los artículos referentes a materno infantil fueron publicados en el 2010; acerca de la historia y filosofía del cuidado, el 40\% fueron publicados en el año 2010.

Fuente: Balseiro L. y Cols. Evaluación interna de la Revista Enfermería Universitaria 2007-2011. México, 2013. ticas diferentes ocho artículos (Cuadro II). En este cuadro llaman poderosamente la atención que fueron publicados en el 2007-2008 y 2010, los artículos publicados de ética y legislación; sin embargo, no existen publicaciones de bioética, ecología y demografía.

La salud laboral de las enfermeras ha sido también un tema poco estudiado, ya que sólo se tienen siete artículos en los cinco años estudiados. Por el contrario, los artículos de mayor publicación han sido de enfermería comunitaria y de administración de los servicios de enfermería, áreas temáticas de apoyo que han tenido una mayor publicación en los años 2010-2011 (Figura 2). En relación con

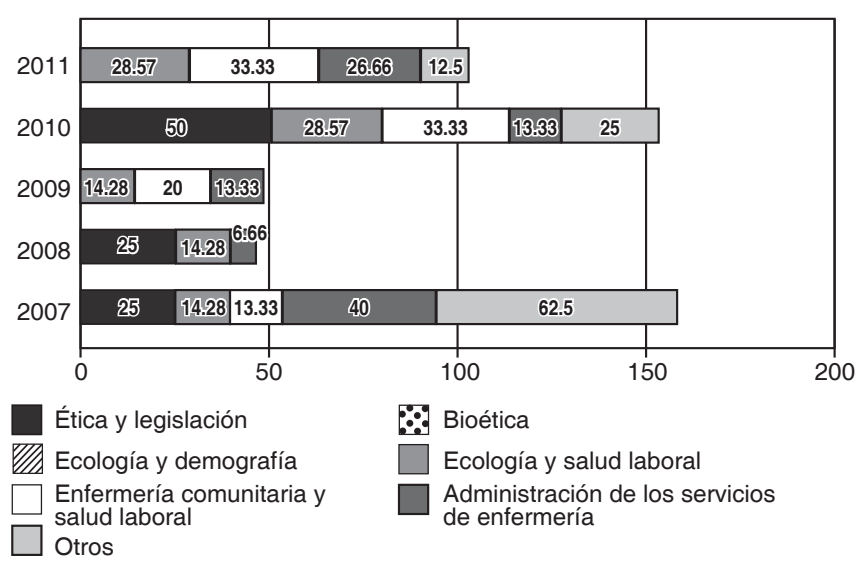

Figura 2. Distribución de las áreas de apoyo y del conocimiento de enfermería.

En la presente figura podemos observar que el $50 \%$ de los artículos sobre ética y legislación se publicaron en la Revista Enfermería Universitaria en el 2010; no existen publicaciones en las temáticas de bioética, ecología y demografía. Hablando del área de ecología y salud laboral, se puede visualizar que el $60 \%$ de los artículos se encuentran publicados en el 2010 y 2011 . En el área de enfermería comunitaria y salud laboral, cerca del $67 \%$ de las publicaciones están ubicadas entre el 2010 y 2011. En lo que se refiriere a administración el $40 \%$ fueron publicados en el año de 2007; asimismo, en otras áreas de apoyo el $62 \%$ aparece en los números de la revista del año 2007.

Fuente: Balseiro L. y Cols. Evaluación interna de la Revista Enfermería Universitaria 2007-2011. México, 2013

Cuadro II. Artículos por áreas de conocimiento de enfermería: áreas de apoyo I.

\begin{tabular}{|c|c|c|c|c|c|c|c|c|c|c|c|c|c|c|}
\hline \multirow[b]{2}{*}{ Año } & \multicolumn{2}{|c|}{$\begin{array}{c}\text { Ética y } \\
\text { legislación }\end{array}$} & \multicolumn{2}{|c|}{ Bioética } & \multicolumn{2}{|c|}{$\begin{array}{l}\text { Ecología y } \\
\text { demografía }\end{array}$} & \multicolumn{2}{|c|}{$\begin{array}{l}\text { Ecología y salud } \\
\text { laboral }\end{array}$} & \multicolumn{2}{|c|}{$\begin{array}{c}\text { Enfermería } \\
\text { comunitaria y salud } \\
\text { laboral }\end{array}$} & \multicolumn{2}{|c|}{$\begin{array}{l}\text { Administración de } \\
\text { los servicios de } \\
\text { enfermería }\end{array}$} & \multicolumn{2}{|c|}{ Otros } \\
\hline & FO & $\%$ & FO & $\%$ & FO & 0 & FO & $\%$ & FO & $\%$ & FO & $\%$ & FO & $\%$ \\
\hline 2007 & 1 & 25 & 0 & 0 & 0 & 0 & 1 & 14.28 & 2 & 13.33 & 6 & 40.00 & 5 & 62.5 \\
\hline 2008 & 1 & 25 & 0 & 0 & 0 & 0 & 1 & 14.28 & 0 & 0 & 1 & 6.66 & 0 & 0 \\
\hline 2009 & 0 & 0 & 0 & 0 & 0 & 0 & 1 & 14.28 & 3 & 20 & 2 & 13.33 & 0 & 0 \\
\hline 2010 & 2 & 50 & 0 & 0 & 0 & 0 & 2 & 28.57 & 5 & 33.33 & 2 & 13.33 & 2 & 25 \\
\hline \multirow[t]{2}{*}{2011} & 0 & 0 & 0 & 0 & 0 & 0 & 2 & 28.57 & 5 & 33.33 & 4 & 26.66 & 1 & 12.5 \\
\hline & 4 & 100 & 0 & 0 & 0 & 0 & 7 & 100 & 15 & 100 & 15 & 100 & 8 & 100 \\
\hline
\end{tabular}

Fuente: Balseiro L. y Cols. Evaluación interna de la Revista Enfermería Universitaria 2007-2011. México, 2013. 
Cuadro III. Artículos por áreas de conocimiento de enfermería: áreas de apoyo II.

\begin{tabular}{|c|c|c|c|c|c|c|c|c|}
\hline \multirow[b]{2}{*}{ Año } & \multicolumn{2}{|c|}{ Salud mental y psiquiatría } & \multicolumn{2}{|c|}{ Microbiología } & \multicolumn{2}{|c|}{ Nutrición y dietética } & \multicolumn{2}{|c|}{ Geronto-geriatría } \\
\hline & FO & $\%$ & FO & $\%$ & FO & $\%$ & FO & $\%$ \\
\hline 2007 & 0 & 0 & 0 & 0 & 0 & 0 & 0 & 0 \\
\hline 2008 & 0 & 0 & 0 & 0 & 0 & 0 & 0 & 0 \\
\hline 2009 & 0 & 0 & 3 & 75 & 0 & 0 & 3 & 100 \\
\hline 2010 & 0 & 0 & 1 & 25 & 0 & 0 & 0 & 0 \\
\hline \multirow[t]{2}{*}{2011} & 0 & 0 & 0 & 0 & 0 & 0 & 0 & 0 \\
\hline & 0 & 0 & 4 & 100 & 0 & 0 & 3 & 100 \\
\hline
\end{tabular}

Fuente: Balseiro L. y Cols. Evaluación interna de la Revista Enfermería Universitaria 2007-2011. México, 2013.

otras áreas de apoyo del conocimiento de enfermería en salud mental y psiquiatría no se publicó ningún artículo en esos años y en temáticas de microbiología sólo hubo cuatro artículos; en nutrición y dietética cero artículos y en geronto-geriatría sólo tres artículos. El panorama que muestran estos resultados da una idea de que estas son temáticas de poco interés de los autores que escriben en la revista (Cuadro III).

\section{DISCUSIÓN}

En cuanto a las áreas de conocimiento de enfermería, la $R e$ vista Enfermería Universitaria publicó 12 artículos relativos a los cuidados básicos, 33 de metodologías de enfermería, 34 de docencia, cero de médico-quirúrgicas, tres de materno infantil y diez de historia del cuidado. Estos datos fueron comparados con los publicados por Pilar Serrano Gallardo y cols., quienes analizaron la productividad científica de la Revista Metas de Enfermería mostrando que en la ciencia de enfermería, las investigaciones son relativamente recientes, las cuales fueron iniciadas hace poco más de una década; se han publicado estudios relativos a las áreas concretas de la enfermería, como el realizado por Barreda y cols., en el área de materno-infantil, o el llevado a cabo por Asiaín y Marín centrado en la enfermería intensiva. También se han publicado estudios sobre la producción enfermera a través de las revistas de mayor difusión: entre éstos se encuentra el realizado por Torra i Bou, que analiza tres publicaciones enfermeras en el trienio 1991-1993. Gálvez Toro y cols., basándose en el análisis de referencias y de citas, llegan a la conclusión de que las enfermeras consultan poco las revistas de enfermería al escribir sus artículos. En relación con las áreas de apoyo de enfermería, la Revista de Enfermería Universitaria en el periodo estudiado, sólo publicó cuatro artículos de las temáticas de ética y legislación, cero de bioética, cero de biología y demografía, siete de ecología y salud laboral, quince de enfermería comunitaria y quince de administración de los servicios. Desde luego, esto representa un reto importante para los autores y la propia revista, dado que es necesario incrementar los autores que escriben artículos relacionados con el conocimiento de enfermería en estas áreas.

\section{CONCLUSIONES}

En cuanto a las áreas del conocimiento de enfermería, la revista publicó 12 artículos relativos a los cuidados básicos, metodología de enfermería, docencia, enfermería infantil e historia del cuidado. No hubo publicaciones del área médico-quirúrgica. En las áreas de apoyo predominan los artículos sobre cuidados básicos y aspectos metodológicos de la profesión, administración en los servicios de enfermería y de enfermería comunitaria, aunque existe una nula productividad en artículos de ética, legislación, ecología y demografía. Aunque la publicación de un artículo para una revista científica constituye un proceso riguroso de revisión, arbitraje y validación para asegurar la calidad y aporte de su cantidad, es menester continuar realizando el esfuerzo de los autores por escribir en las áreas del conocimiento de enfermería poco exploradas, a fin de lograr publicaciones que evidencien y enriquezcan la práctica profesional de enfermería en estos campos.

\section{BIBLIOGRAFÍA}

1. Gálvez T, López IM, Sánchez V, Poyatos E. Evaluación de la actividad científica de la enfermería española. Impacto y aislamiento en el año 2000. Index de Enfermería. 2001; 10 (34): 54-64. Disponible en: http:// bit.ly/1jRwPsn

2. Lorenzini A, Paluccini MH, Goncalves ML, Félix FC, Freitag LM, Itayra $\mathrm{M}$ et al. La evaluación de periódicos científicos qualis y la producción brasileña de artículos del área de enfermería. Rev Latino-am Enfermagen. 2009; 17 (3): 1-9. Disponible en: http://bit.ly/1fHIJj9

3. Serrano G. Análisis de la producción científica de la Revista Metas de Enfermería. Index de Enfermería. 2005; 14 (48): 78-82. Disponible en: http://bit.ly/lhMIQzC

4. Araujo GT, Vieyra JA, Mesa MA. La evaluación de revistas biomédicas mexicanas: una descripción del proceso 2005 para el índice Artemisa. Acta Ortopédica Mexicana. 2009; 23 (6): 366-375. Disponible en: http:// bit.ly/1mVrQLL 
5. Pardo C, Mallebrera E, García MJ, Reolid M, Delicado MV. Características de la Producción científica en enfermería en la década de 19851994. Enfermería Clínica. 2001; 2 (2): 51-63. Disponible en: http://bit. ly/1g0FvMf

6. Sobrido M, Sobrido N, González C, Pichel MJ, García MM, Prieto A. Revistas españolas de enfermería en bases de datos nacionales e internacionales. Index de Enfermería. 2005; 14 (48-49): 74-77. Disponible en: http://bit.ly/lgarAj3

7. Fundación Index. Revistas Núcleo de Enfermería: la elección de la mejor revista para publicar un trabajo. Fundación Index. Disponible en: http:// bit.ly/1i9HUDd

8. Gómez I, Bordons M. Limitaciones en el uso de los indicadores bibliométricos para la evaluación científica. Instituto de Estudios Documentales sobre Ciencia y Tecnología. 2009. Disponible en: http://bit.ly/1lz9HSs 